\title{
Ausgewählte Aspekte der Textsorte autographes Vorwort einer wissenschaftlichen Abhandlung im Deutschen und Polnischen - Versuch einer Konfrontation
}

\begin{abstract}
The purpose of this article has been to compare texts of forewords in Polish and German scientific monographs. The analysis has focused on the following selected aspects: the structure of the forward and its position in relation to other parts of the monograph, the title as well as introductory and closing remarks. It has been discovered that Polish and German authors have a radically different approach to this type of text, though judging by the fact they call their texts forewords, these texts must belong to the same category.
\end{abstract}

Diese Studie soll einen Beitrag zur kontrastiven Fachtextlinguistik im Kommunikationsbereich der Wissenschaften leisten. Seit den 90er Jahren wandte sich die Fachsprachenforschung immer mehr der Textebene der fachlichen Kommunikation zu und versuchte u.a. eine Typologie von Fachtextsorten zu erstellen. Die meisten Analysen beschränkten sich auf Texte, Textsorten, Textmuster in einer Sprache, aber solche Analysen, die die Besonderheit einer Textsorte näher bestimmen, können auch zur Klärung der Unterschiede zwischen den Texten einer Textsorte in zwei oder mehreren Sprachen beitragen.

Obwohl Analysen von Textsorten, auch Fachtextsorten sehr oft durchgeführt werden, sind wissenschaftliche, auch (text)linguistische Beschreibungen der akademisch relevanten Textsorten auch in einer Sprache immer noch ziemlich selten (vgl. z.B. Gläser 1990, Graefen 1997, Trumpp 1998).

Textsorten unterscheiden sich sowohl innerhalb einer Sprache als auch im Vergleich zu anderen Sprachen unter anderem bezüglich ihres Aufbaus, Morphologie (z.B. Tempusgebrauch), Syntax (bevorzugte Satzstrukturen), 
Verwendung unterschiedlicher spezifischer Sprachmittel (z.B. Nominalstil, häufige Verwendung von Funktionsverbgefügen etc.) und durch weitere Merkmale. Dies zu zeigen ist auch das Ziel dieses Aufsatzes.

Der Untersuchung werden Vorworte in den auf deutsch und polnisch verfassten wissenschaftlichen Abhandlungen unterzogen. Hier werden auch von deutschen Nicht-Muttersprachlern verfasste Texte berücksichtigt. Ich bin mir der Gefahr einer solchen Verfahrensweise bewusst, muttersprachliche Interferenzen auf die Textstuktur in die Untersuchung mit einzubeziehen und damit verfälschte Ergebnisse zu erzielen. Ich gehe jedoch von der Überzeugung aus, dass die Texte, die in deutschen und polnischen wissenschaftlich angesehenen Verlagen herausgegeben werden, hochgradig standarisiert sind und eine allgemeine Konvention der jeweiligen Diskursgesellschaft befolgen.

Unten werden Ergebnisse einer kontrastiven Analyse von 100 Vorworten (50 deutschen und 50 polnischen) erörtert. Deren Auswahl war durch das Ziel bestimmt, die Untersuchung möglichst breit anzulegen, da ich der Meinung bin, dass eine empirische Fundierung für die Textlinguistik von größter Bedeutung ist (vgl. Lundquist 1989: 122). Es ist dabei anzumerken, dass die hier präsentierten Ergebnisse als vorläufig aufzufassen sind und keinen Anspruch auf Vollständigkeit erheben. Die Analyse wird im Rahmen einer größeren Projektstudie fortgesetzt.

Das Vorwort einer wissenschaftlichen Abhandlung fungiert nicht als selbstständige Textsorte, sondern als ein Element der umfangreicheren Textsorte Monographie. Eine wissenschaftliche Abhandlung ist eine der vielen Textsorten (z.B. wissenschaftlicher Artikel, Rezension, fachbezogener Essay, Lehrbuch usw.) der fachbezogenen Kommunikation. Sie zeichnet sich durch einen sehr hohen Fachlichkeitsgrad aus, der sich in einer gediegenen Fachkenntnis des Verfassers und des Lesers und in der Verwendung der Fachsprache manifestiert. Als charakteristische Merkmale der Wissenschaftssprache nennt man in der Regel die Präzision des Ausdrucks, Eindeutigkeit und Sachlichkeit.

Eine wissenschaftliche Abhandlung gilt als eine fachinterne Fachtextsor$t^{1}{ }^{1}$, da sie die fachbezogene Kommunikation zwischen den Fachleuten (Wis-

\footnotetext{
1 Gläser (1990) schlägt folgende Klassifizierung der Textsorten innerhalb der Wissenschaftssprache vor:

die Ebene der fachinternen Kommunikation

fachinformationsvermittelnde Textsorten (darunter auch Monographien)

interpersonal / kontaktive Textsorten

direktive Textsorten

didaktisierende Textsorten

die Ebene der fachexternen Kommunikation

didaktisierende Textsorten
} 
senschaftlern) ermöglicht. Sie weist einen hohen Spezialisierungs- und Fachlichkeitsgrad, die auf Seiten der Kommunikationspartner die entsprechende Fachkompetenz voraussetzen und fachliche Kommunikation vermitteln (vgl. Bungarten 1981, Gläser 1990). Eine Monographie weist je nach Zielsetzung und Intention des Verfassers, auch nach dem Wissenschaftszweig eine variable Aufbaustruktur auf. Die Makrostruktur einer Monographie lässt sich folgendermaßen skizzieren:

- Titelblatt

- Inhaltsverzeichnis

- Vorwort des Autors (fakultativ) und / oder Einleitung

- Verzeichnis der Abkürzungen (fakultativ)

- Textkörper

- Anmerkungsapparat (fakultativ)

- Glossar (fakultativ)

- Literaturverzeichnis

- Autoren- und Sachregister (fakultativ).

Ein Vorwort ist nicht obligatorisch und nur bei umfangreicheren Arbeiten üblich. Es wird von Genette (1989: 10) zu sog. Paratexten ${ }^{2}$ gerechnet. Es sind Texte, die dem Haupttext (Hauptwerk) untergeordnet sind. Dank ihnen wird aus einem Text ein Buch. Sie unterscheiden sich voneinander durch ihre Funktionen, die von unterschiedlichen Parametern wie Epoche, Kultur, Autor, Art der Veröffentlichung, Adressatenkreis abhängen.

Cho (2000), der Verfasser der einzigen mir bekannten Abhandlung, die dem Vorwort gewidmet ist, definiert diese Textsorte wie folgt:

Bei Vorworten in wissenschaftlichen Abhandlungen handelt es sich um paratextuelle Elemente, die in wissenschaftichen Texten dem Haupttext vorangestellt sind. Der Vorwortverfasser zielt darauf ab, (i) den Leser über die historische, bibliographische und biographische Entstehungsgeschichte des Werks zu informieren, (ii) Leistungen des Autors bzw. Werte in angemessener Weise anzuerkennen, (iii) begriffliche/thematische Probleme vorwegzunehmen, die er im Haupttext behandeln will, um dadurch den Leser mit der Struktur und dem Inhalt des Haupttextes vertraut zu machen, oder (iv) eine spezifische Beziehung zwischen ihm und einer oder mehreren Personen in der Öffentlichkeit aufzuzeigen sowie aufrechtzuerhalten und herzustellen (Cho 2000: 189).

popularisierende Textsorten

verhaltenssteuernde Textsorten (weiter untergliedert in instruktive und direktive Textsorten).

Andere Klassifizierungen sind durchaus möglich, wie z.B. die von Beneś (1981: 186) postulierte Einteilung, die auf folgenden drei Kriterien basiert: das Medium (geschriebene vs. gesprochene Sprache), der Charakter der Stoffbehandlung (z.B. Bericht, Erörterung usw.) und der Fachlichkeitsgrad und Einstellung zum Leser. Den Kriterien nach ist die Monographie dem sog. Forschungsstil zuzuordnen, im Gegensatz z.B. zu einem Lehrbuch, das sich durch seinen belehrenden Stil auszeichnet.

${ }^{2}$ Als andere paratextuelle Elemente eines Werkes fungieren bei Genette (1989) Titel, Zwischentitel, Inhaltsverzeichnis, Abkürzungstabelle, Motto, Anmerkung, Literaturverzeichnis, Sachverzeichnis, Namenverzeichnis usw. 
In diesem Beitrag lehne ich mich an diese Definition an, wobei mir klar ist, dass sie erweiterungs- und präzisionsbedürftig ist, wenn man z.B. die fließende Grenze zwischen einem Vorwort und einer Einleitung bedenkt, worauf auch in den unten stehenden Ausführungen ${ }^{3}$ kurz eingegangen wird.

Eine grobe Unterteilung der Vorworte kann nach dem Kriterium des Textproduzenten vorgenommen werden. Je nach der Art der Abhandlung werden Vorworte entweder von dem Verfasser der wissenschaftlichen Arbeit selbst geschrieben (sie werden in Anlehnung an Cho (2000: 187) als autograph bezeichnet) oder sie begleiten den Haupttext eines anderen Autors. Sie werden z.B. von einem Herausgeber einer wissenschaftlichen Reihe oder dem Betreuer des Autors angefertigt. Solche Vorworte werden allograph (vgl. Cho 2000: 187) genannt.

Im Folgenden konzentriere ich mich ausschließlich auf autographe Vorworte.

Bei der in diesem Beitrag dargestellten kontrastiven Analyse wird in erster Linie auf strukturelle Aspekte der Textsorte eingegangen. Die strukturelle Realisierung der Texte einer Textsorte wird u.a. mit Hilfe folgender Merkmale (vgl. Cho 2000: 171ff.) charakterisiert:

- Textumfang und seine Gliederung (Teiltexte bzw. Absätze und ihre inhaltlichen Beziehungen);

- Handlungsstruktur des Textes (Anordnung und Hierarchie der im Text vollzogenen Handlungen, bzw. Handlungstypen und -sequenzen);

- Gliederungssignale im Text;

- Sprachliche Objektivierung der Texte (textsortenspezifische Sprachmittel, z.B. morphologische, syntaktische, lexikalische, phraseologische, stilistische)

Wenn man Vorworte in wissenschaftlichen Arbeiten einer Analyse unterzieht, scheint mir noch ein weiteres Phänomen von großer Relevanz zu sein, nämlich die Stellung des Vorworts in der gesamten Abhandlung und sein Verhältnis zu anderen Teilen der Monographie. Dies wird auch in dieser Studie berücksichtigt.

Es werden zwei Aspekte eines Vorworts unter die Lupe genommen, der eine, der zu den textexternen Merkmalen betrachtet gerechnet werden kann - die Platzierung der Texte in der Abhandlung, ihre Eìngliederung in die Abhandlung, und der andere, der eine textinterne Eigenschaft darstellt, nämlich der strukturelle Aspekt, der den Textumfang und seine Gliederung, d.h. die inhaltlichen Beziehungen zwischen den einzelnen Textabschnitten, umfasst.

${ }^{3}$ Näheres zu der Problematik siehe: Beata Mikolajczyk, Vorwort - Einleitung aus textlinguistischer Sicht. In: Studia Germanica Posnaniensia. - in Vorbereitung. 


\section{1. ÜBERSCHRIFT, PLATZIERUNG UND EINBEZIEHUNG IN DAS INHALTSVERZEICHNIS}

Die analysierten polnischen und deutschen Texte sind mit folgenden Überschriften versehen:

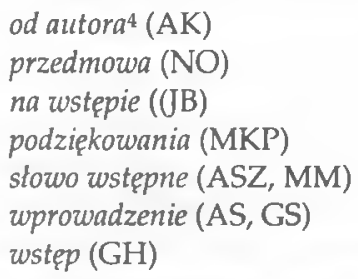

\section{Vorwort}

\author{
Einführung $(\mathrm{DH})^{5}$
}

Vorwort (KF)

\section{Vorwort zur ersten Auflage + Vorwort zur zweiten Auflage ${ }^{6}$}

\section{Danksagung + Einleitung}

Das Vorwort eröffnet fast jede wissenschaftliche Abhandlung. Obwohl oft behauptet wird, dass das Vorwort nicht zu den Pflichtteilen einer wissenschaftlichen Arbeit gehört, ergreifen die Autoren die Gelegenheit, im Vorwort den Kontakt mit ihren Lesern aufzunehmen und sie in die Lektüre ihres Werkes einzuführen.

Das Vorwort (oder alle dieser Textsorte angehörenden Texte) werden in der Regel in den polnischen Werken dem Inhaltsverzeichnis nachgestellt und im Inhaltsverzeichnis berücksichtigt. (Die Tatschache, ob ein Teil der Arbeit im Inhaltsverzeichnis genannt wird, ist wichtig, denn Inhaltsverzeichnisse sagen z.B. etwas über die Gewichtung von Teilen der Abhandlung und ihre Relation zueinander aus.) Die meisten Verfasser der auf Polnisch erschienenen Werke gliedern es jedoch nicht in den gesamten Text der Abhandlung ein, und nummerieren das Vorwort nicht ( $90 \%$ aller im Korpus vorhandenen Fälle). In vereinzelten Fällen (10\% der analysierten Abhandlungen) wird das Vorwort als erstes Kapitel der Monographie angesehen und mit der entsprechenden Nummer versehen, (z.B. MM), betitelt wird es mit dem Begriff Vorwort (z.B. słowo wstępne) Nur sehr selten werden einfüh-

4 Die vorgeschlagenen Übersetzungsäquivalente: od autora - Vorwort des Verfassers; przedmowa - Vorwort; na wstępie - zum Geleit; podziękowania - Danksagung; stowo wstępne - Vorwort; wprowadzenie - Einführung, Einleitung; wstęp - Vorwort.

${ }^{5}$ Cho (2000) nennt noch andere Überschriften Vorbemerkung, zum Geleit, Geleit, Dank, Danksagung, Widmung. Sie traten in den von mir analysierten Monographien nicht auf.

${ }^{6}$ Die Kombination von zwei Vorworten zur ersten und zur zweiten Auflage wird aus meinen Überlegungen ausgeklammert. Auch das Zusammentreffen (ein äußerst seltener Fall) von einem allographen und einem autographen Vorwort in einer wissenschaftlichen Arbeit wird hier nicht weiter untersucht. 
rende Bemerkungen nicht nur als das erste Kapitel behandelt, sondern mit einer eigenen Überschrift versehen, z.B. Rozdziat 1. Jezzyk angielski w reklamie. Uwagi wstępne. (WC/JS) oder 1. Na wstępie (JB). In meinen Recherchen bin auf keine polnische Abhandlung gestoßen, in der das Vorwort dem Inhaltsverzeichnis vorangestellt wird und aus diesem Grunde auch da nicht vorhanden ist.

Auch in den auf Deutsch geschriebenen Monographien ist das Vorwort in der Regel nach dem Inhaltsverzeichnis platziert und in das Inhaltsverzeichnis aufgenommen, aber es wird nicht nummeriert. Gelegentlich wird es zusätzlich von den anderen Abschnitten der Arbeit abgesondert, indem seine Seiten im Gegensatz zu allen anderen Kapiteln (sowohl im Inhaltsverzeichnis als auch im Text) mit römischen Ziffern markiert werden (z.B. KF). Dies geschieht auch, wenn das Vorwort nicht in die Struktur der Arbeit eingegliedert wird (die Überschrift wird nicht nummeriert) und zusammen mit der Einleitung (dieser Teil wird nummeriert) nach dem Inhaltsverzeichnis steht (z.B. WY).

Die meisten, deutschsprachigen bis jetzt analysierten Abhandlungen verfügen sowohl über ein Vorwort / eine Danksagung als auch eine Einleitung. In solchen Fällen befindet sich die Danksagung oder das Vorwort vor dem Inhaltsverzeichnis, seine Überschrift wird nicht nummeriert. Es wird also als ein gesonderter Teil der Arbeit angesehen. Die Einleitung dagegen wird nach dem Inhaltsverzeichnis platziert und als ein integraler Abschnitt der Arbeit (mit der eigenen Nummer, in der Regel 1, gelegentlich 0) betrachtet.

\section{ERÖFFNUNG - MOTTO}

Die Autoren versehen manchmal ihre Vorworte mit einem Motto, dies geschieht jedoch in den polnischen Texten viel häufiger (16 von 50 Vorworten) als in den deutschen (nur einmal belegt), z.B.:

"Ich hasse die Leute, die rasch Systeme bauen, und ich werde dazu sehen, daß meines sich nie ganz schließt" (Canettt, E. (1973): Die Provinz des Menschen, München, 127) (HHL)

Rozmyślając nad kłamstwem zdałam sobie sprawę z tego, jak często my, językoznawcy, analizujemy z przyjemnościq zdobywców słowa wypowiadane, a jak często ze skrupulatnościq empiryków nie zauważamy tego, co przernilczane i czego nie można poddać "skrupulatnej analizie gramatycznej", gdyż na powierzchni struktury językowej nie znajdujemy żadnych gramatycznych wyktadników. Innymi stowy zdalam sobie sprawę z tego, ze gramatyk wprawdzie "wie wszystko" o strukturze jezyka, niewiele jednak może powiedzié,, jak ludzie się rzeczywiście porozumiewają. Jolanta Antas (1999: 8) (MM)

Es muss bemerkt werden, dass auch deutsche Autoren sich gern eines Mottos bedienen, sie gebrauchen es aber in der Einleitung nicht im Vorwort.

Dies ist ein Indiz dafür, dass die deutschen und polnischen Verfasser das Vorwort unterschiedlich behandeln, für polnische Autoren gehört das Vorwort als ein vorbereitender Abschnitt zum Hauptteil der Arbeit und seine 
Aufgabe besteht darin, gedanklich in die Arbeit einzuführen und auf die zentrale Problem-/Fragestellung und die wesentlichen Gedankengänge hinzuweisen. Ein Motto kann die Einbettung der gewählten Fragestellung in ein weiteres Umfeld oder den Rahmen einer Fachdiskussion gewährleisten. Die meisten deutschen Autoren sehen es anders und in ihren Abhandlungen gilt die Einleitung als der Teil, der z.B. Zielsetzungen und Arbeitshypothesen, historische Voraussetzungen, Fragestellungen eröffnet.

\section{ABSCHLUSS}

Unten werden abschließende Elemente geschildert, die in einem Vorwort angewandt werden.

Ort, Datum, Vor- und Zuname des Verfassers

Aleksander Kiklewicz Olsztyn, luty 2006 (AK) Helsinki, Juni 1978 Merkku Moilanen (MMD1) Wrocław, jesień 2006 Tomasz Matysek (TM) Münster, im Frühjahr 1976 Manfred Kohrt (MK)

Ort, Datum, Autor

Kraków, wrzesień 1990r. Autor (ASZ)

Vor- und Familienname des Verfassers

Datum, Vor- und Zuname des Verfassers September 1978 Josef Häfele (JH)

Ort, Datum, Initialbuchstaben des Vor- und Nachnamens des Autors

Nijmegen, Oktober $1981 \quad$ W.K. (WLK)

Gosheim, im August 1998

H.H.L. (HHL)

Marek Kochan (MKP)

Ort, Datum

Milwaukee, WI - Warszawa - Poznań - Augsburg, im Oktober 1983 (FKB)

Zielona Góra - Niwiska, 1999-2003

(GS)

Keine Angaben

Einerseits machen sich bestimmte Asymmetrien im Gebrauch einiger Abschlusskonventionen bemerkbar, andererseits zeichnen sich gravierende statistische Unterschiede ab. Während die meisten polnischen Autoren $(87 \%)$ ihre Vorworte ohne jegliche Angaben beenden, unterzeichnet die überwiegende Mehrheit aller deutschen Verfasser einer Monographie (92\%) ihren Paratext und versieht ihn noch mit Ort und Datum der Abfassung. Dies ist ein Indiz dafür, dass polnische Wissenschaftler diesen Teil ihrer Arbeit als einen integralen Teil der Abhandlung ansehen und die deutschen Forscher das Vorwort nicht als einen Bestandteil der eigentlichen Arbeit betrachten. 


\section{INHALTSSTRUKTUR}

Sogar in Ratgebern für Studierende, wie man eine wissenschaftliche Arbeit abfassen soll, wird sehr oft die These vertreten, dass man im Vorwort lediglich auf äußere Umstände (z.B. Materialbeschaffung, Zugang zu Originalen) bei der Abfassung der Arbeit eingehen und für allfällige Hilfeleistung danken kann. Es soll jetzt geprüft werden, welche Inhalte Vorworte in den auf Polnisch und auf Deutsch veröffentlichten wissenschaftlichen Werken enthalten. Dabei wird zwischen Vorworten, die allein (d.h. ohne Einleitung) vor dem Haupttext platziert sein und Vorworten, die in Begleitung einer Einleitung erscheinen.

\subsection{Vorwort, das als der einzige vorangestellte Paratext gilt}

\section{Einführung in die behandelte Thematik}

Jednym z największych sukcesów zrywów spolecznych w Niemczech w 1848 było jak sądzili początkowo wspólcześni zniesienie cenzury. W rzeczywistości spoleczne i polityczne wydarzenia związane z Wiosną Ludów wyznaczyły ważną cenzurę w ksztaltowaniu się w państwach niemieckich nowych mechanizmów kontroli. Wtedy to rozpocząl się proces przejścia od systemu cenzury prewencyjnej do systemu cenzury represyjnej. Decydujący wpływ na kształtowanie się tego ostatniego miały $w$ Prusach ustawy $\mathrm{i}$ rozporządzenia prasowe $z$ lat 1849-1851, a ostatecznym jego usankcjonowaniem byla ustawa prasowa $\mathrm{z} 1874$ obowiązująca na terenie całej Rzeszy Niemieckiej. (MR)
Simone de Beauvoirs Feststellung, die Frau werde gedacht als das andere, das zweite und zweitrangige Geschlecht, das unter dem Begriff des Menschen nicht voll subsumierbar sei, hat bis heute nicht an Gültigkeit verloren. Nach wie vor ist diese Vorstellung der ideologische Überbau eines hierarchischen Geschlechterverhältnisses, dessen Fortbestand sie sichert, insofern sie nicht nur das Fremdbild, sondern das Selbstbild der Frau reflektiert. Diese Arbeit geht der Frage nach, wie diese Vorstellung sprachlich - durch sprachliche Interaktion und deren Wahmehmung sowie durch geltende Sprachnormen - konstituiert und reproduziert wird. (KF)

\section{Entstehungsgeschichte}

To miała być zupełnie inna książka. Gdy jechałem do Stanów Zjednoczonych jesienią 2002 roku, mialem ambitny zamiar napisania historii amerykańskiej filozofii polityki po roku 1945. Zamieszkałem wraz $\mathrm{z}$ rodziną $\mathrm{w}$ mieście Union City (...).Nie była to moja pierwsza wizyta w Kalifornii. (...) Nie było wątpliwości: znalazłem się w środowisku wielokulturowym. (...) Postanowiłem więc tym razem rozpocząć swoje badania amerykańskiej filozofii polityki
Bei dem Versuch, eine aktualisierte 2. Auflage meiner "Stilistik der deutschen Sprache" von 1986 zu schreiben, kam ich seit dem Beginn 1996/97 immer mehr vom Wege ab: Der zeitliche Abstand war zu groß, auf dem Feld inzwischen zu Vieles geschehen. Geblieben sind die Kapitel 1. die neu verbundenen Kapitel 1.2. uns 1.4 von 1986. Das Übrige zielt auf eine Textstilistik des Deutschen, von Spillner $(1997,253)$ als Desiderat bezeichnet. Bezüge zur Rhe- 
od sprawy wielokulturowości. (...) Książka ta powstała więc $w$ wyniku fascynacji światem, którego nie znamy. (ASZ1) torik konnte ich nur stellenweise andeuten; sie genauer auszuloten wäre sicher ein Gewinn. (BS)

\section{Motivationen des Autors}

Pisząc książkę pt. Akty deprecjonizujace siebie $i$ innych. Studium pragmalingwistyczne szukałam odpowiedzi na pytanie, na czym polega mechanizm deprecjacji z punktu widzenia analizy lingwistycznej oraz co warunkuje jego skuteczność. (MM)

\section{Schwerpunkte und / oder Begriffsbestimmung}

Przez samą deprecjację rozumiem takie zachowanie językowe i niejęzykowe, świadome bądź mimowolne, które ma na celu zagrożenie pozytywnemu aspektowi twarzy interlokutora, a szczególnie stanowi atak na jego pozytywnie zintegrowany obraz samego siebie. Należy więc deprecjacja do klasy aktów mowy określanych mianem "Aktów Zagrażania Twarzy" (Face Threatening Acts) (...) Innymi słowy, interesowało mnie zbadanie tekstotwórczych warunków deprecjacji, a następnie odpowiedź na pytanie: jak to się dzieje, że wypowiedź zupełnie neutralna jest przez odbiorcę odbierana jako umniejszająca, a czasem wypowiedź jawnie deprecjonująca okazuje się niefortunna $w$ swym działaniu. (MM)
Die Stilistik, die sich mit Texten befasst, ist vielerlei Hinsicht durch traditionelle Denkweise (Typisierung, con Stilelementen, Stilistik als Erbe der Elocutio) geprägt, aber auch behindert. Demgegenüber hat die Gesprächsstilistik einschließlich Soziolinguistik bis hin zur Standardsprachenforschung und zu sozialen Stilen, auch zur Erforschung von mündlicher Kommunikation in Institutionen und feministischer Linguistik, Prosodieforschung und interaktionaler Stilistik, viel deutlichere Fortschritte auf dem Gebiet der Stilistik gemacht. (BS)

\section{Stand der Forschung}

Takie ujęcie wydaje mi się szczególnie ważne i potrzebne, bo po pierwsze otworzy szerokie, nowe na polskim gruncie, pole badawcze (...) mechanizmy umniejszania w większości prac rozpatrywane były od strony psychologicznej. Analizy językoznawcze, jakie znaleźć można w opracowaniach m.in. szkoły Pało Alto czy Friedmana Schulza von Thuna, były pobieżne i miały charakter li tylko wspomagający techniki terapeutyczne. (MM)
Bisher vorliegende linguistische Arbeiten zu diesem Gegenstandsbereich suchen entweder zu belegen, daß Frauen in gegengeschlechtlichen Interaktionen von Männern dominiert und unterdrückt werden, oder setzen sich mit dem sexistischen Charakter "der Sprache" bzw. einzelner Sprachnormen auseinander. Trotz ihres feministischen Erkenntnisinteresses ist beiden Forschungsrichtungen letztlich eine rein sprachwissenschaftliche bzw. konversationsanalytische Perspektive immanent. Dies führt dazu, daß die Autorinnen einerseits bei einer - empirisch unterhaltbaren - qualitativen Argumentation verharren 
(Männer beanspruchen mehr Redezeit, unterbrechen häufiger ect.) und andererseits nur am Rande die Frage behandeln, welches gesellschaftliche "Wissen" und welche Wahrnehmungsmuster dem Individuum mit sexistischen Sprachnormen übermittelt werden. Ein inhaltlicher Zusammenhang zwischen den beiden Forschungsrichtungen wird so nicht hergestellt. (KS)

\section{Materialauswahl}

Podstawą mojego naukowego warsztatu była przede wszystkim analiza materiałowa. Sposób zbierania materiału egzemplifikacyjnego w praktyce okazał się niezwykle trudnym dla lingwisty, dyskusyjnym i dyskutowanym problemem. (...) Korzystalam z materiałów zarejestrowanych na taśmie wideo $w$ trakcie warsztatów prowadzonych przez p. Antas w Klubie "Pod Jaszczurami" pt. Sztuka rozmawiania, a także zgromadzonych na zajęciach prowadzonych na III roku dziennikarstwa UJ. (MM)
Ich werde ganz überwiegend Schriftexte zum Thema machen, habe auch versucht, von gesprächs-stilistischer Forschung dafür zu profitieren. (BS)

Viele der bearbeiteten Beispiele wurden mit Studierenden der Universität des Saarlandes bearbeitet, andere verdanke ich den Studierenden, teils weil sie sie als Materialien in Seminarsitzungen einbrachten, teils stammen sie aus Seminar- und Abschlussarbeiten, andere wurden mir nach dem Examen gebracht oder sogar geschickt. (BS)

\section{Darstellung der Gliederung des Textes}

Praca zasadniczo składa się z dwóch części: teoretyczno-metodologicznej, w której między innymi opisałam elementy zdarzenia językowego konieczne przy badaniu takich zjawisk, jakim jest deprecjacja. (...) W części teoretycznej opisałam też taki typ nadawcy, u którego deprecjacja jest stylem komunikacyjnym, oraz wspomniałam o możliwych perlokucyjnych reakcjach odbiorcy po usłyszeniu umniejszającego komunikatu. W części materiałowej przeanalizowalam bogaty materiał, opisując sam mechanizm deprecjacji $w$ warstwie tekstotwórczej, oraz próbowałam pokazać, kiedy te mechanizmy nabierają mocy pragmatycznej i są komunikacyjnie skuteczne. Składa się ona $z$ trzech części. (MM)

\section{Methodisches Vorgehen}

Analizując zagadnienia deprecjacji, przez cały czas balansowałam między metodo- 
logią kognitywną a pragmatyką. Przykladowo w pierwszym projekcie pracy używałam szerzej Langackerowskich wymiarów obrazowania, przede wszystkim odwoływałam się do pojęcia subiektywizacji tta kotwiczqcego. W trakcie analiz jednak świadomie się z tego wycofałam, pozostawiając jedynie Langackerowskie pojęcie "sceny", gdyż niezwykle szczegółowe i drobiazgowe analizy kognitywne - w moim przekonaniu - zatarłyby przejrzystość całego wywodu. (MM)

\section{Adressatenkreis}

Praca ta przeznaczona jest dla nauczycieli języków obcych i wszystkich osób związanych $\mathrm{z}$ ich edukacją na uniwersytetach i w kolegiach językowych oraz w ośrodkach realizujących wszelkie formy ksztalcenia podyplomowego, a także dla studentów i doktorantów kierunków neofilologicznych. (MW)

\section{(manuskript)technische Informationen}

W książce wykorzystałem fragmenty swoich wcześniej opublikowanych artykułów: Ideologia wielokulturowośći: kontekst filozoficzny $i$ polityczny, "Ergo" 2003, nr 6; (ASZ1)
Ich habe mich bemüht, die neue Rechtschreibung zu nutzen, das gilt auch für Zitate aus der Sekundärliteratur, nicht aber für die Beispieltexte. Die linguistischen Auffassungen, die für die Beschreibungen genutzt werden, sind jeweils knapp dargestellt, weil das Wissen darum in der Regel nicht gleichmäßig verteil ist. (BS)

\section{Danksagung}

Jest takie chińskie przysłowie: „Nauczyciel otwiera drzwi, ale wejść przez nie uczeń musi sam". Chciałam podziękować Pani Prof. Jolancie Antas za niezwykle piękny i ważny dla mnie komfort poszukiwania prawdy. (...) Dziękuję także Pani Prof. Elżbiecie Tabakowskiej za - mam nadzieję, że w tym kontekście nie zabrzmi to zbyt familiarnie - bycie "dobrym aniolem stróżem", zawsze otwartym i gotowym do pomocy. Wierzę, że zanadto tej serdeczności Pani Profesor nie wykorzystałam, choć czasem pokusa była we mnie
Danken möchte ich auch Thea und Theodor Frank meinen Eltern, die mir mit moralischer Unterstützung, lebensklugen Rat, Geld, Tiefkühlkost und Keksen in einem sehr "ganzheitlichen" Sinne geholfen haben. (KF) 
silniejsza. Dziękuję za wsparcie, wskazania naukowe i nie tylko. Dla mnie to byl niekwestionowany zaszczyt. (MM)

\section{Widmung}

Swoją książkę chciałabym zadedykować mojemu mężowi Robertowi, który nigdy nie pozwolił mi zapomnieć, że celem wszelkiego poznania jest drugi człowiek, a nasza praca ma o tyle sens, o ile zbliża nas do innych ludzi. (MM)
Widmen möchte ich diese Arbeit "G + $\mathrm{C}^{2 \prime \prime}$, die mir den Rücken stärkten, wenn der Atem ausgehen wollte, die Lichter setzten, wenn ich im Kreise lief, die sich mit mir freuten, wenn eine Hürde genommen war. (BW)

Die oben dargestellten Asymmetrien bestätigen die schon vorhin gezogenen Schlüsse. Auch bei der Wahl der erörterten Inhalte verfahren deutsche und polnische Forscher anders. In den polnischen Vorworten werden solche Themen angesprochen, die sich direkt auf die wissenschaftliche Problematik beziehen, d.h., es erfolgt eine Zielorientierung und Motivierung und die Einbindung in das wissenschaftliche Umfeld. Die deutschen Vorworte dagegen enthalten in überwiegender Mehrheit Inhalte, die keinen direkten Bezug auf die im Textkern diskutierte Thematik aufweisen.

Neben dem Inhalt lassen sich auch große Unterschiede im Umfang der Vorworte feststellen. Die deutschen Paratexte umfassen eine, max. zwei Seiten, polnische Vorworte sind in dieser Hinsicht viel unterschiedlicher, sie umfassen von zwei bis 13 Seiten. Der Durchschnittsumfang beträgt 6-9 Seiten.

\subsection{Vorwort, das in Kombination mit einem anderen Paratext auftritt}

Bei der Kombination der Texte Vorwort + Einleitung 7 , was im untersuchten Korpus der in der deutschen Sprache verfassten Abhandlungen die häufigste Vorgehensweise der Verfasser ( $85 \%$ der analysierten Monographien) darstellt, kommt es zu einer Differenzierung der Inhalte, die in den Texten besprochen werden. Auch der Umfang der beiden differiert entscheidend. Das Vorwort ist viel kürzer, es umfasst in der Regel eine bis zwei Seiten. Der Umfang der Einleitung ${ }^{8}$ reicht von einem ganz kurzen (eine knappe Seite - in den 95\% aller Abhandlungen dieses Typus) bis zu längeren Texten (z.B. 25 Seiten bei WY). Ein solches gemeinsames Auftreten von zwei vorangestellten Paratexten ist in polnischen wissenschaftlichen Arbeiten äußerst selten.

7 Auf die Kombination przedmowa + wstẹp bin ich nur in einer polnischen Abhandlung gestoßen, sie scheint auch für polnische wissenschaftliche Arbeiten außergewöhnlich zu sein., deswegen wird sie in meine Erwägung nicht mit einbezogan.

${ }^{8}$ Auf die Struktur der Einleitung wird in dieser Studie aus Platzgründen nicht eingegangen. 


\section{Vorwort + Einleitung}

Diesen Typ ist nur in den auf Deutsch verfassten Abhandlungen anzutreffen. Das Vorwort beschränkt sich in einem solchen Fall auf:

\section{Information,}

- dass die Monographie auf Grund einer Dissertation' / Habilitationsschrift bearbeitet wurde

Die vorliegende Studie ist die leicht überarbeitete Fassung meiner Dissertation, die im Wintersemester 1989/90 vom Fachbereich für Philosophie und Sozialwissenschaften der Technischen Universität Braunschweig angenommen wurde. (WY)

- welche Veränderungen seitens des Verfassers bei der Überarbeitung vorgenommen wurden

Mit der Bereitstellung der Ergebnisse für eine breitere Öffentlichkeit ergab sich die Notwendigkeit, theoretischen Ballast abzuwerfen, um die Arbeit auch dem praktisch interessierten Leser zugänglich zu machen - sei es dem Journalisten, der sich intensiver mit der Interviewpraxis auseinandersetzt, oder dem Lehrer, der im Unterricht Texte aus der Massenkommunikation analysiert oder allgemeine Strukturen von Dialogen erarbeitet. (...) Das Manuskript wurde (...) für die Veröffentlichung in verschiedenen Passagen überarbeitet und gekürzt. (RRH)

- von wem der Verfasser angeregt wurde, sich mit der Thematik zu befassen

Die Anregung zur Problemstellung dieser Arbeit verdanke ich Prof. Werner. Zunächst war daran gedacht, eine Typologie der Zeichenbildung im Deutschen mit zunehmenden Komplikationsgraden zu erstellen und an Beispielen zu demonstrieren., eine Thematik, die Prof. Werner in seinen Publikationen und Lehrveranstaltungen immer wieder beschäftigte. Viele Gesichtspunkte, die er in Vorlesungen und Seminaren behandelte, sind in die Arbeit eingegangen. Im Laufe der eigenen Untersuchungen wurde diese ursprüngliche Anordnung und Thematik modifiziert. (EW)

\section{Einführung in die behandelte Thematik}

Die vorliegende Arbeit versteht sich als ein interdisziplinärer Beitrag zur Linguistik und Literaturwissenschaft, in dem Methoden und Ergebnisse der Sprechakttheorie diskutiert und auf ihre Nutzbarmachung für die Analyse und Interpretation von Dramen hin untersucht werden. In ihrer Funktion als ein methodischer Beitrag zur Drameninterpretation zielt die Studie auf einen zentralen Untersuchungsaspekt dieser Gattung, nämlich auf das Verhältnis von Sprechen und Handeln im Drama.

Die Sinnfälligkeit von Analysekriterien aus der Sprechakttheorie für eine derartige Untersuchung liegt nahe, ist jedoch in der bisherigen Forschung nur in wenigen Aufsätzen und keineswegs immer befriedigend geleistet worden. Auf der Basis einer ausführlichen Diskussion zentraler sprechakttheoretischer Problembereiche wird eine systematische und zugleich exemplarische Anwendung durchgeführt und versucht, die Relevanz der Sprechakttheorie für die Analyse und Interpretation des Dramas zu demonstrieren. (RS)

${ }^{9}$ Eine solche Information findet man auch in der Danksagung, die einleitenden Gedanken vorausgeht, z.B. bei BH. 


\section{Zielsetzung}

Dieses Buch setzt sich zum Ziel, die verschiedenen textlinguistischen und pragmalinguistischen Forschungsaktivitäten zu erfassen und sie in übergeordnete systematische und wissenschaftstheoretische Zusammenhänge zu stellen; es will somit die gegenseitigen Bezüge der Richtungen wie auch der erlangten Erkenntnisse verdeutlichen und den inneren Zusammenhalt der Disziplin erhellen. Die "Orientierung zur Textlinguistik" möchte es also leisten, den Begriff ,Textlinguistik' inhaltlich zu erfassen und ihn in seinem Umfang zugehöriger und zuständiger Richtungen möglichst vollständig darzustellen. (HK)

\section{Adressatenkreis}

Aus meinen Ausführungen dürfte klar hervorgehen, daß diese Monographie sowohl für den Sprachtheoretiker als auch für den Didaktiker sowie für in- und ausländische Germanistikstudenten, Sprachliebhaber und Lehrbuchautoren von großem Interesse ist. (JD)

\section{Materialauswahl}

Im europäischen Kulturraum bieten die Bibelübersetzungen den am besten dafür geeigneten Korpusbereich. Meine Wahl fiel dabei auf die Paulusbriefe, die neben sprach- und übersetzungswissenschaftlicher Fragestellungen auch interessante kulturwissenschaftliche Einblicke in zwei Sprachkulturen, die jüdische und die hellenistische, erlauben. (UT)

\section{Anwendungsmöglichkeiten des gewählten Ansatzes und / oder Hinweise für den Leser}

Der methodische Ansatz, auf der Grundlage der außersprachlichen Bedingungen das sprachliche Handeln zu prognostizieren, kann prinzipiell auf andere Formen von Sprechtätigkeit übertragen werden.

Gerade von diesem Hintergrund kommt die theoretische Absicherung gegenüber der Darstellung der Methodik und der Untersuchungsergebnisse zu kurz. Für den in dieser Hinsicht interessierten Leser sind allerdings im zweiten Kapitel, das Hinweise zur Entwicklung der Methodik enthält, einige theoretische Grundpositionen näher erläutert. Auch dem praxisorientierten Leser wird damit entgegengekommen - das stärker sprachwissenschaftlich ausgerichtete zweite Kapitel kann durchaus vernachlässigt werden zugunsten einer Erörterung der Rahmenbedingungen politischer Fernsehinterviews im ersten Kapitel, die dann über die Untersuchungshypothesen unmittelbar in das dritte Kapitel mit der eigentlichen Analyse überleitet. (RRH)

\section{(manuskript)technische Hinweise}

Zur Zitiergepflogenheit: Eine Notierung wie (1976[1968]) bedeutet, daß der angeführte Beitrag 1968 veröffentlicht wurde, hier aber nach einer Ausgabe, Sammlung oder Quelle von 1976 zitiert wird. (HK)

\section{Danksagung}

Ich danke Prof. Hennig und Prof. Rehbein, den beiden Gutachtern der Dissertation, sehr herzlich für wertvolle Anregungen und weiterführende Kritik zur Abfassung des Manuskripts sowie für ihre kontinuierliche Diskussionsbereitschaft. Insbesondere Prof. Hennig hat mich immer wieder in meinem Forchungsinteresse bestärkt. Wichtige Hinweise verdanke ich auch den intensiven Diskussionen mit Sven Sager. (UM)

\section{Widmung}

Dem Knirps, der inzwischen auf die Welt kam, ist die Arbeit gewidmet. Möge ihm etwas von seinem zuversichtlichen Lachen bleiben. (FKB) 
Oft wird behauptet, dass zwischen einem Vorwort und einer Einleitung streng zu unterscheiden ist (z.B. Genette 1989: 157).

- Ein Vorwort dient dem Zweck, situative oder umstandsbedingte Kenntnisse im Hinblick auf historische oder institutionelle Hintergründe der Entstehung des Werkes zu übermitteln;

- Eine Einleitung dient dem Zweck, fachliche Kenntnisse zu übermitteln, und zwar im Hinblick auf architektonische Fragen des Textes, themabedingte Probleme im allgemeinen oder im Hinblick auf begriffliche Erklärungen. (Cho 2000: 176)

An diese Anweisungen halten sich die meisten Autoren nicht. In den hier analysierten Vorworten findet man auch Äußerungen, die laut Genette (1989) für Einleitungen typisch sind. D.h., auch in den wissenschaftlichen Abhandlungen, deren Verfasser den Entschluss gefasst haben, ihrer Arbeit sowohl ein Vorwort als auch eine Einleitung voranzustellen, werden die beiten Textsorten zuweilen nicht klar unterschieden, was den deutschpolnischen Vergleich noch erschwert.

Wissenschaftliche Arbeiten mit anspruchsvollem Inhalt sollen auch in ihrer formalen Struktur bestimmten Richtlinien entsprechen. Das garantiert, dass der Inhalt auch effizient vermittelt wird.

Deutsche Autoren von Publikationen, die sich mit der Struktur der wissenschaftlichen Arbeiten auseinandersetzten, schlagen Folgendes vor:

Man verwechsle nicht Vorwort und Einleitung. Die Einleitung ist ein Teil der Arbeit, das Vorwort dagegen gehört nur mittelbar zur Arbeit. Es ist sozusagen ein Begleitbrief, den der Verfasser seiner Arbeit mit auf den Weg gibt, um darin Erklärungen über die Arbeit abzugeben. Das Vorwort kann daher Persönliches enthalten, was die Einleitung als Teil der Arbeit nicht kann. (Standop 1994: 24)

Die oben präsentierte Analyse kann die These nicht hundertprozentig bestätigen; für die meisten deutschen Autoren scheint die Grenze zwischen Vorwort und Einleitung verschwommen zu sein. Im Vorwort nennt der Verfasser die Ziele und die Veranlassung zu seinem Buch. Dort gibt es Hinweise auf vorige Auflagen, und es wird Dank abgestattet an Ratgeber und Helfer; oft gibt es an dieser Stelle Hinweise auf andere Wissenschaftler, die man schon kennt oder die besser bekannt sind. Des Weiteren gibt er Auskunft über seine wissenschaftliche Methode und die allgemeine Lage der Literatur zu seinem Thema. Es können auch weitere Punkte ausgeführt werden wie: persönlicher Anlass der Arbeit, Rechtfertigung der Themenstellung, Einordnung des Themas in den aktuellen Diskussionsstand/ Geschichte und Stand der Forschung, formale und methodologische Legitimation, Kriterien der Materialauswahl, eigene Begriffsbildungen, Vorstellung von Zielsetzungen und Arbeitshypothesen, Überblick über den Aufbau und die Argumentationsfolge. Im Vorwort kann der Verfasser zusätzlich die emotionale Seite seiner Arbeit zum Ausdruck bringen. 
In den polnischen Arbeiten wird das Vorwort in den meisten Fällen mit der Einleitung gleichgesetzt (darauf weisen auch unterschiedliche polnische Überschriften der Paratexte hin) und enthält allerlei Informationen die sowohl für das Vorwort als für die Einleitung einer deutschen Abhandlung charakteristisch sind.

Es war zwar nicht das Ziel dieses Beitrags, Kriterien zu nennen, nach denen man zwischen der Textsorte Vorwort und Einleitung in einer wissenschaftlichen Abhandlung unterschieden könnte, aber es kann festgehalten werden, dass anhand der oben erarbeiteten Aspekte der Texte es unmöglich ist, eine Reihe signifikanter textsortenspezifischer Merkmale zu ermitteln und über Oppositionsbildungen $\mathrm{zu}$ einer Unterscheidung dieser zwei Textsorten zu gelangen, diese betrifft sowohl deutsche als auch polnische Monographien. Bei der Behandlung von polnischen Texten ist dieses Bedürfnis, ein Inventar solcher Kriterien zu ermitteln, auch dringend, um feststellen zu können, ob in der polnischen Wissenschafts-sprache zwischen diesen Textsorten unterschieden wird.

\section{LITERATURVERZEICHNIS}

Beneš, Eduard, 1981. Die formale Struktur der wissenschaftlichen Fachsprache in syntaktischer Hinsicht. In: Theo Bungarten (Hrsg.), Wissenschaftssprache. Beiträge zur Methodologie, theoretische Fundierung und Deskription. München: Fink Verlag: 185-212.

Brinker, Klaus, 1997. Linguistische Textanalyse. Eine Einführung in Grundbegriffe und Methoden, 4., durchgesehene und ergänzte Aufl. Berlin: Erich Schmidt Verlag.

Bungarten, Theo, 1981. Wissenschaft, Sprache und Gesellschaft. In: Theo Bungarten (Hrsg.), Wissenschaftssprache. Beiträge zur Methodologie, theoretische Fundierung und Deskription. München: Fink Verlag: 14-53.

Cho, Kuk-Hyun, 2000. Kommunikation und Textherstellung: Studien zum sprechakttheoretischen und funktional-kommunikativen Handlungskonzept; mit einer handlungsfundierten Untersuchung der Textsorte Vorwort in wissenschaftlichen. Münster: Dissertation der Philosophischen Fakultät der Westfälischen Wilhelms-Universität Münster.

Genette, Gérard, 1989. Paratexte. Das Buch vom Beiwerk des Buches. Frankfurt a.M./New York: Peter Lang Verlag.

Gläser, Rosemarie, 1990. Fachtextsorten im Englischen. Tübingen: Gunter Narr Verlag.

Graefen, Gabriele, 1997. Der Wissenschaftliche Artikel - Textart und Textorganisation Frankfurt a.M./Berlin/Bern/New York: Peter Lang Verlag.

Gühlich, Elisabeth/Raible, Wolfgang, 1977. Linguistische Textmodelle. Grundlagen und Möglichkeiten. München: Fink Verlag.

Hoffmann, Lothar, 1988. Vom Fachwort zum Fachtext. Beiträge zur angewandten Linguistik. Tübingen: Gunter Narr Verlag.

Hoffmann, Lothar (Hrsg.), 1990. Empfehlung - Standard - Norm. Beiträge zur Rationalisierung in der Fachkommunikation. Leipzig: Bibliographisches Institut.

Lundquist, Lita, 1989. Coherence in scientific texts. In: W. Heyndrich et al. (Hrsg.), Connexity and coherence. Berlin: de Gruyter Verlag: 122-149.

Standop, Ewald 1994, Die Form der wissenschaftlichen Arbeit. Heidelberg u. Wiesbaden: Quelle u. Meyer.

Trumpp, Eva Cassandra, 1998, Fachtextsorten kontrastiv. Englisch - Deutsch - Französisch. Tübingen: Gunter Narr Verlag. 
Verzeichnis der analysierten, in diesem Aufsatz erwähnten Texte (mit Auflösung der Siglen)

Vorworte in den auf Polnisch abgefassten Monographien

AK - Kiklewicz, Aleksander, 2006, Język, komunikacja, wiedza. Mińsk: WTAA Prawa i ekonomika.

AS -Skibiński, Adam, 2003, Homo significus. Autorozprawa o poznaniu-języku. Warszawa: Uroboros.

ASZ -Szulc, Aleksander, 1991, Historia języka niemieckiego. Warszawa: PWN.

ASZ1 - Szahaj, Andrzej, 2004, E pluribus unum? Dylematy wielokulturowości i politycznej poprawności. Kraków: Universitas.

JB - Bralczyk, Jerzy, 2004, Jezzyk na sprzedaż czyli o tym, jak język stuży reklamie i jak reklama używa języka. Gdańsk: Gdańskie Wydawnictwo Psychologiczne.

GS -Skommer, Grzegorz, 2006, Wyrażenia porównawcze. Model struktury i semantyki porównań $w$ jezzyku polskim i norweskim. Poznań: Wydawnictwo Naukowe UAM.

MKP - Kochan, Marek, 2005, Slogany $w$ reklamie i polityce. Warszawa: Wydawnictwo TRIO.

MM - Majewska, Małgorzata, 2005, Akty deprecjonujące siebie $i$ innych. Studium pragmalingwistyczne. Krak6w: Universitas.

MR - Rajch, Marek, 2004, Cenzura pruska w Wielkopolsce w latach 1848-1918. Poznań: Wydawnictwo Poznańskie.

MW - Wysocka, Maria, 2003, Profesjonalizm w nauczaniu języków obcych. Katowice: Wydawnictwo Uniwersytetu Śląskiego.

NO -Ostrowski, Norbert, 2006, Studia z historii czasownika litewskiego. Iteratiwa. Denominatiwa. Poznań: Wydawnictwo Naukowe UAM.

TM - Matysek, Tomasz, 2007, Literatura niemiecka w teorii $i$ praktyce. Wroclaw: Oficyna Wydawnicza ATUT.

WC/JŚ - Chłopicki, Wladyslaw / Świątek, Jerzy, 2000, Angielski w polskiej reklamie. Warszawa: Wydawnictwo Naukowe PWN.

Vorworte in den deutschsprachigen wissenschaftlichen Arbeiten

BS -Sandig, Barbara, 2006, Textlinguistik des Deutschen. Berlin / New York: Walter de Gruyter Verlag.

BW -Wotjak, Barbara, 1992, Verbale Phraseolexeme in System und Text. Tübingen: Max Niemeyer Verlag.

DH - Handl, Daniela, 2003, HotFlow und StAdT: Sprechakt-theoretische Konzepte für die dynamische Steuerung und Unterstützung dokumentbasierter Geschäftsvorgänge. Darmstadt: Dissertation der Technischen Universität.

EW -Weigand, Edda, 1978, Die Zuordnung von Ausdruck und Inhalt bei den grammatischen Kategorien des Deutschen. Tübingen: Max Niemeyer Verlag.

FKB - Keller-Bauer, Friedrich, 1984, Metaphorisches Verstehen. Eine linguistische Rekonstruktion metaphorischer Kommunikation. Tübingen: Max Niemeyer Verlag.

HHL - Lüger, Heinz-Helmut, 1999, Satzwertige Phraseologismen: eine pragmalingiustische Untersuchung. Wien: Verlag Edition Praesens.

HK -Kalverkämper, Hartwig, 1981, Orientierung zur Textlinguistik. Tübingen: Max Niemeyer Verlag.

KF - Frank, Karsta, 1992, Sprachgewalt: Die sprachliche Reproduktion der Geschlechterhierarchie. Elemente einer feministischen Linguistik im Kontext sozialwissenschaftlicher Frauenforschung. Tübingen: Max Niemeyer Verlag.

MK -Kohrt, Manfred, 1976, Koordinationsreduktion und Verbstellung in einer generativen Grammatik des Deutschen. Tübingen: Max Niemeyer Verlag. 
MMD1 - Moilanen, Markku, 1979, Statische lokative Präpositionen im heutigen Deutsch. Wahrheits- und Gebrauchsbedingungen. Tübingen: Max Niemeyer Verlag. RRH - Hoffmann, Rolf-Rüdiger, 1982, Politische Fernsehinteroiews. Eine empirische Analyse sprachlichen Handelns. Tübingen: Max Niemeyer Verlag.

RS -Schmatenberg, Reinhard, 1982, Sprechakttheorie und dramatischer Dialog. Ein Methodenansatz zur Drameninterpretation. Tübingen: Max Niemeyer Verlag.

TK -Kotschi, Thomas, 1974, Probleme der Beschreibung lexikalischer Strukturen. Untersuchungen am Beispiel des französischen Verb. Tübingen: Max Niemeyer Verlag.

UT - Topczewska, Ursula, 2004, Phraseolexeme in Paulusbriefen und ihre Wiedergabe im Deutschen und Polnischen anhand ausgewählter Bibelübersetzungen. Trier: WVT Wissenschaftlicher Verlag.

WLK - Lessen Kloeke van, Wus, 1982, Deutsche Phonologie und Morphologie. Merkmale der Markiertheit. Tübingen: Max Niemeyer Verlag.

WY - Yang, Wenliang, 1990, Anglizismen im Deutschen. Am Beispiel des Nachrichtenmagazins "Der Spiegel". Tübingen: Max Niemeyer Verlag. 